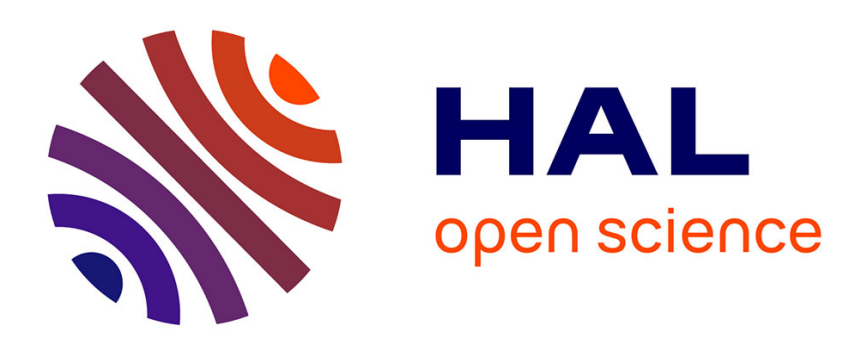

\title{
Visualizing alternative scenarios of evolution in heritage architecture
}

Iwona Dudek, Jean-Yves Blaise

\section{To cite this version:}

Iwona Dudek, Jean-Yves Blaise. Visualizing alternative scenarios of evolution in heritage architecture. i-KNOW '11 - 11th International Conference on Knowledge Management and Knowledge Technologie, Sep 2011, Graz, Austria. pp.Article No. 45, 10.1145/2024288.2024342 . halshs-01494671

\section{HAL Id: halshs-01494671 https://shs.hal.science/halshs-01494671}

Submitted on 23 Mar 2017

HAL is a multi-disciplinary open access archive for the deposit and dissemination of scientific research documents, whether they are published or not. The documents may come from teaching and research institutions in France or abroad, or from public or private research centers.
L'archive ouverte pluridisciplinaire HAL, est destinée au dépôt et à la diffusion de documents scientifiques de niveau recherche, publiés ou non, émanant des établissements d'enseignement et de recherche français ou étrangers, des laboratoires publics ou privés. 


\section{Visualizing alternative scenarios of evolution in heritage architecture.}

\author{
Iwona Dudek \\ MAP - CNRS \\ 184 av de Luminy \\ 13288 Marseille Cedex 09 (F) \\ $+33491827170$ \\ idu@map.archi.fr
}

\author{
Jean-Yves Blaise \\ MAP - CNRS \\ 184 av de Luminy \\ 13288 Marseille Cedex 09 (F) \\ $+33491827170$ \\ jyb@map.archi.fr
}

\begin{abstract}
Our objective is to support reasoning tasks in heritage architecture with graphics enabling analysts to visualise and share their understanding of how, from a given set of information, alternative scenarios or evolution can be inferred. The paper comments on the nature of the cognitive processes in historical sciences, and on factors that need to be weighed when interpreting sets of information. Visual solutions are proposed, and illustrated on real cases in Kraków Poland. They help spotting where alternative explanations should be considered in order to avoid unjustified assumptions and certitudes on the evolution of artefacts. The contribution expects to demonstrate that reasoning on uncertainties in historical sciences can be fruitfully backed up by concepts and practices from the InfoVis community.
\end{abstract}

\section{Categories and Subject Descriptors H.3.3, H.5.2, J.5, H.1, H.2.5, H.2.8, H.3.1}

\section{General Terms}

Documentation, Reliability, Human Factors, Theory.

\section{Keywords}

Architectural heritage, knowledge modelling, Spatio-temporal data, uncertainty.

\section{INTRODUCTION}

Analysts who investigate on the evolution of architectural artefacts - may it be for research, teaching or protection purposes - need to carry out reasoning tasks in the context of what Gershon identified as "imperfect knowledge" [1]. Our intent is to show that a shared understanding of spatial dynamics, in heritage architecture, can be usefully supported by analytical graphics provided these graphics do take into consideration the nature of the data handled, and the nature of the analyst's cognitive process.

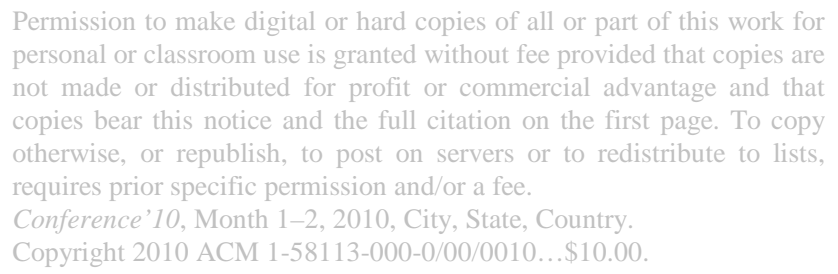

Generally speaking, investigations into the chronology of artefacts imply an interpretation and a cross-examination of heterogeneous, questionable sets of information from which are derived consistent clues and ultimately a scenario of evolution. Naturally a number of solutions do exist to lay out results of such analyses (may they be time-oriented - timelines, time series, etc.; or spaceoriented-GIS platforms, 3D simulations) like [2], [3] or [4] for instance. In a previous contribution [5], we showed that beyond depicting a researcher's conclusions in terms of chronology or of physical changes, visual solutions can also underline processes of change, and support non-verbal thinking.

But in these contributions what is done is choosing a scenario of change, and making it as understandable as possible. Casual and/or teleological reasoning is present, but limited by the scenario chosen. Delattre [6] usefully warns analysts about possible consequences of neglecting to assess choices made, and focusing on interpreted material: "Through our ignorance [of alternative explanations] slogans are inserted in our understanding that, by dint of being repeated, bring to the fore certainties that our knowledge alone would not justify". In this research our objective is to try and support visually the early phases of analysis, before a "most likely" univocal scenario is chosen, before a subjective, "preferred" scenario is spotted and exploited. This implies giving ourselves means to consider, represent, and make visible what E.R. Tufte called alternative explanations [7]. As will be shown in section 3, these alternative explanations exist due to the various interpretations an analyst needs to do of a given set of information. And when the time has come to visualize and share an understanding of how a given artefact has evolved through time, analysts usually rely on the nature of ethnic languages to mention where and why doubts remain, using expression like "a refurbishing may have taken place as a consequence of a fire that supposedly damaged a neighbouring artefact”.

When the analyst is studying one and only one artefact, and when he is the only one to study it, well hesitations are duly passed on to the reader, and it could just as well remain as it is. But when wanting to compare levels of knowledge across artefacts, across historical periods, when trying to understand and cross-examine different interpretations of a given set of information by different analysts, how can we weigh and visualize, in a more or less objective manner, doubts and choices? Should we interpret expressions like "may have occurred" and "it is possible that" as meaning the same thing (not mentioning, naturally, translation problems)? As an answer, we do not intend to over-interpret divergent interpretations (see Figure 1). 

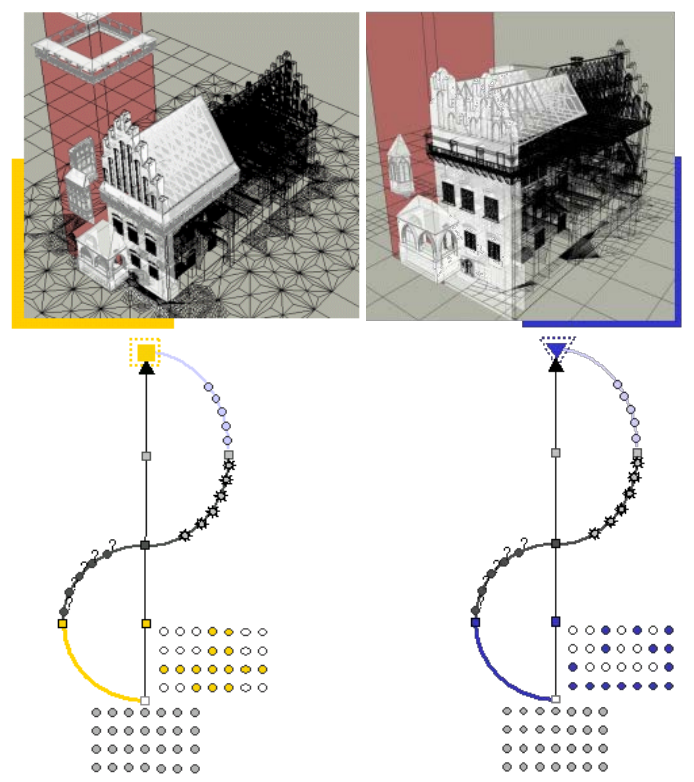

Figure 1. Creation of a reconstruction - (subjective) choice of an information sub-set, casual or teleological argumentation from which a possible spatial layout is proposed, and a unique scenario chosen. Shown here two layouts corresponding to alternative selections inside the information sets on Kraków's old town hall. (From left to right: reconstructions by F. Christ - 1950, and A. Essenwein - 1869) [8].

Instead, we try to understand the nature of the analyst's cognitive process, in order to propose a set of visual tools in charge of delivering an indication about doubts and alternative scenarios. These tools are designed to allow an objective comparison of the scenarios researchers may have privileged, and of their relative levels of complexity. In other words, we shall try to answer to a simple question: can we substitute to verbal figures such as "it is likely that" by a framework for the visualisation of alternative scenarios?

Expected result is to better identify and weigh the uncertainty when handling historical clues, and to uncover patterns of uncertainty within the collection - an application of J. Albert's " $1+1=3$ or more" principle [7]. The approach is tested on the evolution of 27 artefacts located on the market square in Kraków, Poland (see Figure 2). This market square, or Rynek Główny, is a $200 * 200 \mathrm{~m}$ urban space dedicated to trade and administration of the town since its foundation in 1257. Artefacts we analyse are quite well documented thanks to comprehensive historical investigations ever since the 19th century. But they have been built, modified, and for most of them destroyed, over a rather long period - leaving a number of questions unanswered.

In this paper we first analyse factors that need to be weighed by analysts when facing historical evidence in architecture. We then introduce the modelling choices made in order to structure outputs of the information interpretation step (notions of states and transitions, classification of changes occurring during the transition phase, differentiation between confirmed and unconfirmed alternatives, etc.). We then present a set of visual tools designed to support reasoning tasks as well as knowledge sharing in the early phases of architectural analysis. Finally, we briefly report on the implementation and evaluation, in section 5 , and sum up our findings in section 6 .

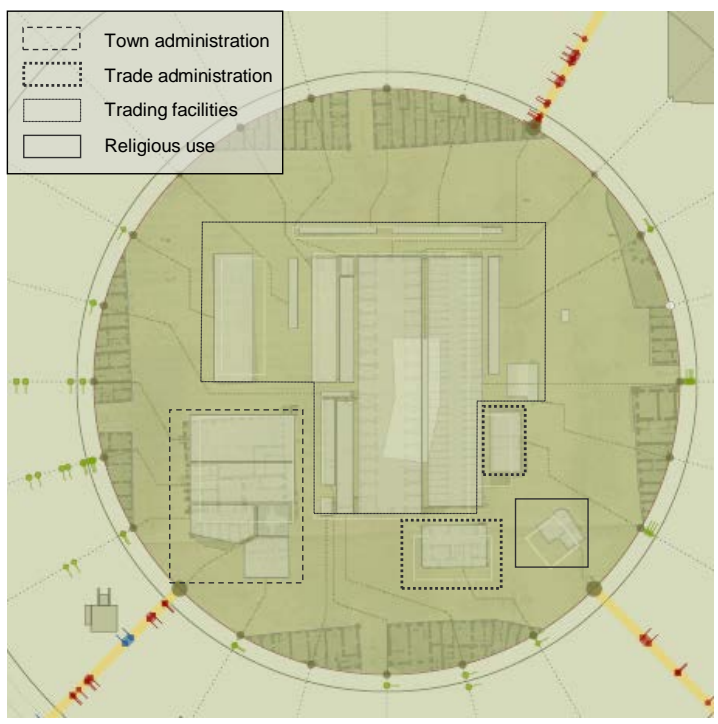

Figure2. Krakow's Main Square with simplified outlines superimposed on an 18th century map and identification of social uses of artefacts.

\section{RESEARCH CONTEXT}

Understanding and providing models to handle the dynamics of change, has been and remains a hot research topic in geography or geospatial sciences. Applications range for instance from the analysis of human movements [9] to the visualisation of physical phenomena [10]. Among models for spatial analysis that [11] usefully compiles, Hagerstrand's time-geography offers a potentially fruitful conceptual framework, clearly described and exemplified in [12]. Time-geography puts space and time on equal terms, and introduces in spatial analyses individual behaviours which are points we also need to make. However these various approaches focus on the modelling of dynamics that have little to do with the very nature of data sets handled in historic sciences (uncertainties, incompleteness, etc.). They neither imply an interpretation of the underlying data sets, nor call for an assessment of reliability of sources. Furthermore, even when dealing with urban changes (see for instance [13]), most approaches use a systematic spatial clustering that cannot be transferred (without losses in semantics) to ill-defined architectural spaces. The issue we are facing closely resembles what [13] identifies as the drawback of "descriptive models [...] based on static situation": a weak understanding of processes and of causal relations.

As mentioned in [5], little has specifically been done, in the field of the architectural heritage, in order to describe and represent visually the time-chain between successive states or moments in the evolution of artefacts. When seen from closer, our research question raises two families of question:

- understanding the analyst's cognitive process, i.e. steps and inputs from raw data to a scenario of evolution,

- supporting this process with relevant graphics, i.e. graphics that amplify cognition [14] on the successive choices the analyst faces when trying to spot and describe artefact changes.

Reasoning into a historical phenomenon, and here into artefact changes, has been described as hypo-deductive by [4] or reductive and non-inductive by [15]. Figure 3 sums up and exemplifies these visions on our application field. 


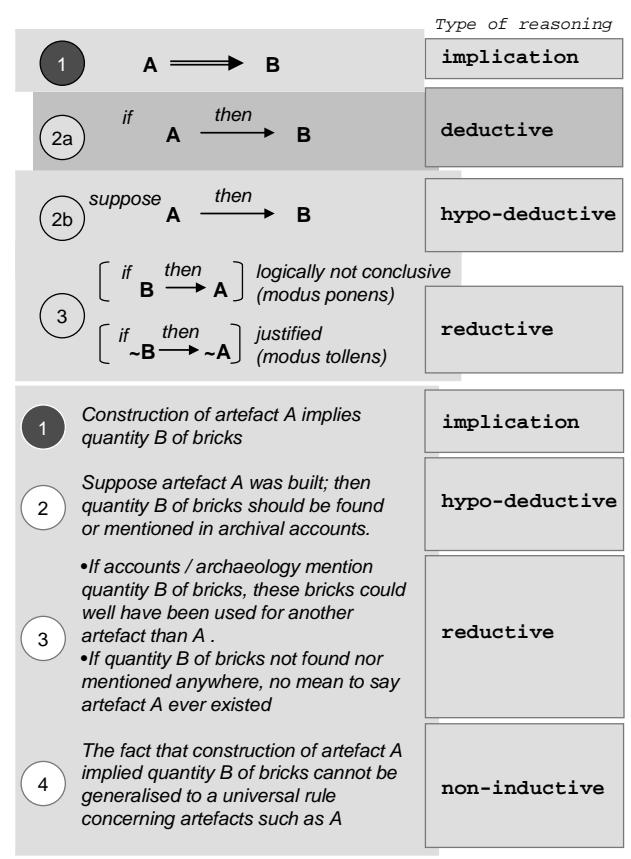

Figure 3. Top, reasoning tasks in historical sciences, based on [15]; bottom, attempts to exemplify his view on classic cases found in architectural heritage analysis.

The point to stress here is that the input an analyst handlesquestionable pieces of information - needs to be interpreted. Each interpretation, willingly or even unwillingly, depends of an a priori knowledge, or paraphrasing [16], of how knowledge interferes with reasoning. This interpretation phase is the moment when alternative choices are offered - and where we expect to provide analytical graphics. Naturally, interpreting pieces of information is not specific to architectural analysis, or more generally to historical sciences. [17] proposes a significant contribution on what uncertainty is, and how to deal with it in the context of intelligence analysis. Authors analyse efforts made in developing classifications and representations for different types of uncertainties in geographic information science on one hand, in the InfoVis community on the other hand. In section 3 we briefly comment on different types of uncertainties in our application field. What is interesting to point out is that although authors do mention that "analysts who have consistent, comprehensive, representations for multiple uncertainties [...] make better decisions", they propose a typology but seemingly no visualisation - a sign that graphics are not that trivial to design... In line with Bertin [18], we consider that graphics are not an end but a mean, including in understanding doubts (see [8]).

Michael Friendly‘s "brief history of data visualization" [19] shows that a wide number of solutions have been investigated on how to represent spatial dynamics. Contemporary research works often privilege representations relying on (and limited by) the GIS paradigm. J.K Rod [20] defines visualisation as been opposed to communication in the fact that the former reveals unknowns, whereas the latter presents knowns. His point of view clearly better matches our approach: provide visual tools aimed at uncovering alternative explanations. We therefore consider heritage architecture analysts need to further investigate approaches developed in the InfoVis community - either in terms of approach, with reference examples in [19] [7], or of inspiring applications like [9][21][22].

\section{NATURE OF THE INFORMATION AND MODELLING CHOICES}

In most publications in and around archaeology and history of architecture pieces of information come at one moment or another to be portrayed as being uncertain, imprecise, unevenly distributed in space and time, etc. Doubt, in a broad sense, is inherent to the information handled. Alternative choices can be made in order to perform reasoning tasks on architectural changes because of the various interpretations a researcher can do of the sets of information available. In historical sciences, researchers base on three types of inputs: general pieces of knowledge (theory, geo-historical knowledge of the artefact under scrutiny and of the area around, analogies, etc.), historical sources (textual or graphic) and finally archaeological investigations. Each in a different manner, these inputs need to be questioned notably in terms of credibility, accuracy and relevance, when analysing the evolution of an artefact. Our position is that although doubts exist in any research where historical inputs are dominant, the nature of the doubts and the way they can be dealt with and visualized, strongly depend on the nature of the information, and on the goals of its interpretation (a view confirmed in [17]). So when talking specifically about historical artefacts analysis, pieces of information must be weighed with regards to at least six factors:

Likelihood: pieces of information are used to identify events, facts that supposedly occurred at time $\boldsymbol{t}$. But is the info enough to consider the fact really established? Phrasing like "event may have occurred" is common in historical reports, and the researcher has to take some decision on how to handle it.

Spatial span: pieces of information can affect the artefact under scrutiny, but also some of its sub-parts, neighbouring artefacts, the whole area around. If some info is duly established for artefact $\boldsymbol{A}$, to which extent should it be taken into consideration when analysing its neighbour artefact $\boldsymbol{B}$ ? This of course depends on the nature of the info: a fire on $\boldsymbol{A}$ is likely to have consequences on $\boldsymbol{B}$, but a change of owner may probably not have any. This factor includes the various uses of analogical reasoning.

Credibility: pieces of information can be transmitted by direct witnesses of an event or fact - but they are often much more indirect, processed several times. We here face a whole gradation from trustworthy contemporary observers to basic hearsays.

Precision: Time slots are classic examples of how pieces of information vary in terms of precision. Historical records may well use phrasings like "at the end of the twelfth century, during the spring period" leaving researchers rather confused at implementation time. It is important to mention that lacks of precision can also occur in spatial data - in our case study an artefact called "Smatruz" (an open market hosting temporary traders) is rather well documented in terms of social use and of chronology, but at this stage impossible to locate.

Transferability: Among the pieces of information researchers deal with are a number of bills, lists of items, inventories, tax reports, etc. These documents do not give us direct indications on the shape and size of the artefact (and not always about its use). For instance, a carpenter's invoice can indicate some change has occurred - but what change? In other words the role of the interpretation is sometimes to transfer the information from one point of view -accounting - to another one - architectural analysis.

Duration: It is almost self-evident to try and differentiate punctual events from processes (and also rather easy to spot a fire, a flood, a bombing as punctual events). However it is in a number 
of cases less obvious to say which is which: is a siege a punctual event or a process? Researchers usually need to identify the temporal granularity that specifically fits their case study, and then can consider events as the smallest time unit, and processes as overlapping several units. Furthermore, researchers welcome pieces of information that can directly feed the analysis of anthropogenic changes. Unfortunately numerous indications relate to long-term processes that are not anthropogenic: if a record tells us an artefact is "in poor condition", it indicates that a long-term process is going on, and little can be inferred on when the process started. These factors are taken into consideration in order to feed the subjective cognitive process that will lead researchers to interpret the information and deduce from it a scenario. But if we want to reason on the whole cognitive process (and not only on the clues we chose to privilege), then we need to visualise all the steps taken, all the choices made. At this point, it is important to remember that what we want to depict is the evolution of an artefact. And the above mentioned factors are used to weigh pieces of information, not artefacts. Some kind of mapping of these factors into concepts and variables portraying artefact changes needs to be done. The following list sums up the notions we have developed up to here:

- We differentiate an artefact's evolution (overall lifetime) from its life cycles (switches from visible to concealed existence).

- We identify transitions - corresponding to changes of the artefact - as opposed to states - periods of stability, corresponding to results of transitions.

- Transitions are classified in general purpose categories - each transition may lead to morphological, structural or functional changes. The impact on the artefact is described by introducing a specific list of features for each category, with varying variable types [23].

- Transitions also need to be analysed with regards to their relation to time, in order to differentiate key steps in the artefact's evolution from regular, "everyday” changes. We propose a hierarchy (with as initial division line key morphological transformations vs episodic transformations) that in addition differentiates positive from negative changes.

- Uncertainty in dating represents a measure (through a lexical scale) of our confidence in the dating of events/processes.

- Confirmed alternatives (disjunctions) represent divergent options on what did occur, basing on indications that are duly established and related to the artefact under scrutiny.

- Unconfirmed alternatives correspond to indications that may be taken into consideration or may be not - when the information is unconfirmed, or more often when it is not directly related to the artefact under scrutiny but to a neighbour (or any semantic group it may belong to).

\section{THE VISUALISATION STEP}

In [5] we introduced a description framework that focuses on the way artefacts get transformed, with a grid of notions giving the analysts the means to date, describe and order events/facts/elements of context that we consider meaningful in the understanding of the artefact's transformations. As a result, the analyst is given a set of graphics called chronographs, composed of three different visualizations (Figure 4):

- Diachrograms present the evolution of an artefact along a time axis. They rely on the classic concept of timeline with markers that position transitions, states, and causality assessments along the time scale.
- Variograms further detail the nature of the artefact's transformation by combining in a parallel visualisation the three categories of changes.

- Features visualisation disposals that foster comparisons across features

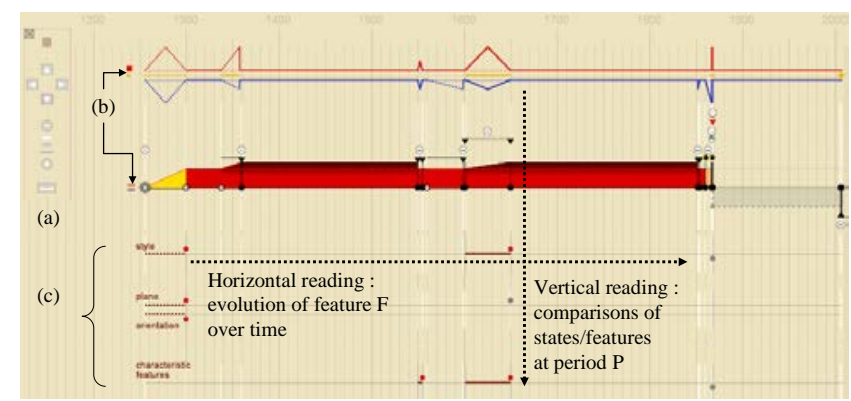

Figure. 4. Top, variogram, middle, diachrogram, bottom, features chronology (partial view) with (a to c) Jscript nested interactive commands of the SVG implementation allowing basic on/off switches and zooming.

The "chronographs" solution has proven quite efficient in performing reasoning tasks - it does help assessing causal relations and orders in time sequences, events and consequences in a robust manner. But a number of weaknesses were also spotted. As mentioned before it corresponds to one and only one scenario of evolution - in other words it does not identify those key time slots when the analyst chooses to favour an interpretation of sources over others. Moreover although doubts about the dating are duly visualised, no mean is given to say that the very existence/relevance of an event is questionable. Finally, comparisons between chronographs are limited by the size of the visualisation - no visual solution has been proposed to consider the collection of artefacts as a whole.

As an answer, we propose a new set of visualisations that capitalize on the user's a-priori understanding of the timeline paradigm, but introduce visual interruptions marking key choices the analyst has to make before adopting an unambiguous scenario (section 4.1). A complementary solution, more or less inspired by the "small multiples" principle (concept attributed to Scheiner's 1626 representation of changes in sunspots over time [19]), is proposed to visualise the collection of artefacts as a whole and uncover patterns of uncertainty in space or in time (section 4.2).

\subsection{Visualising alternative scenarios of evolution of an artefact}

Multi-hypothesis chronology diagrams deliver a quick, synthetic view on where alternatives scenarios exist (in time) and underline options the analysts face. From these diagrams observations and inferences can be made on relative durations, intensities or densities of events, acceleration or deceleration patterns, etc. Various overlapping patterns can also be questioned or formalised for instance using Allen's relations [4].

Multi-hypothesis chronology diagrams reuse categories of changes developed for the chronographs experiment (Figure 5). They localise states (number inside circles) and transitions (red or blue dots and lines) all along a real-scale timeline. Uncertainty in the dating is represented through outline and filling options. A thick yellow line stands for the overall evolution of the artefact. The line is interrupted every time a doubt on how to interpret sources appears. 


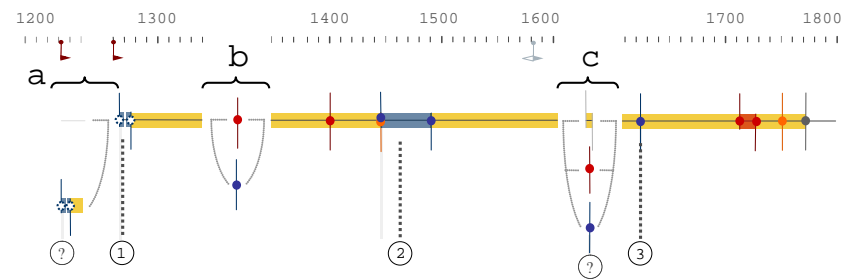

Figure. 5. An indication of periods when alternative scenarios are possible (dating on the time scale), and on the nature of the options: $a)$ there may have been a construction before the first one; $b$ ) either a functional change or a morphological change; c) options are either nothing, or a functional change, or even a morphological change.

Time is still represented in real scale, helping the analyst to weigh durations and densities, and to localise periods of doubt. But because numbers of interruptions may vary from artefact to artefact, lengths of timelines cease to vary according to the sole duration of existence parameter.

Multi-hypothesis chronology diagrams show what options one can think out when interpreting sources, but they do not differentiate confirmed alternatives from unconfirmed alternatives. This is done through graphs of potential interactions, a linear diagrammatic representation along which successive states, transitions, alternatives are presented with brief texts summarising the underlying information (Figure 6).

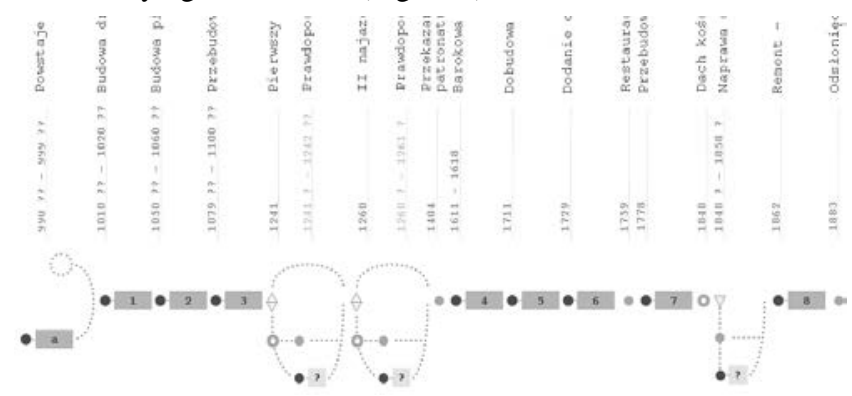

Figure. 6. Graph of potential interactions for St Adalbert's church. A main horizontal line, composed of circles (transitions) and rectangles (states) is interrupted by triangles (confirmed and unconfirmed alternatives). The path from rectangle 7 to rectangle 8 should be read like this: the event or process (vertical text) did occur for sure (triangle downwards) but either it has had limited consequences (light grey circle) or it lead to a morphological change (black circle, rectangle with question mark). The path from rectangle 3 to rectangle 4 is composed of two successive alternatives that should be read like this: the event or process (vertical text) may not have occurred at all (triangle upwards, dotted line straight to next step) but if it did occur (triangle downwards) then ... (back to the same situation as path from rectangle 7 to rectangle 8 ).

As in the previous graphics, a horizontal line (alignment of circles -transitions- and of rectangles - states) standing for the overall evolution of the artefact is interrupted when alternative interpretations of the information are possible. When the information cannot be disambiguated, and alternatives should be considered, circles on the horizontal line are replaced by triangles from which different paths can be taken. Contextual information (i.e. information that cannot be connected to a given change but that may help understanding some other aspect of the artefact's history) can also be integrated (Table 1).

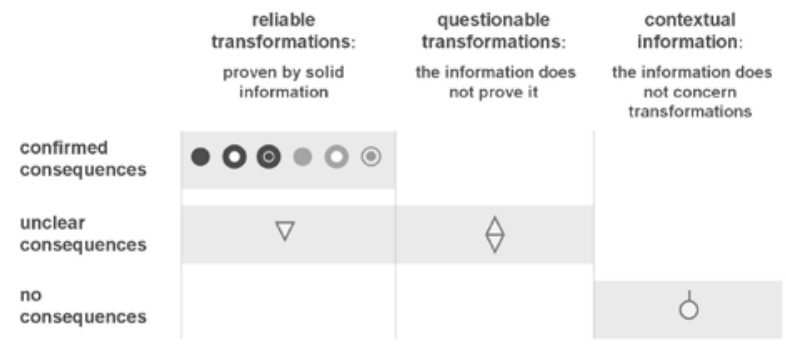

Table 1 Relations of symbols to (columns) nature of the info and (lines) possible consequences.

Differentiation between confirmed alternatives and unconfirmed alternatives is visible in the appearance of the triangle, as well as in the number of possible paths. In order to lower the visualisation's cognitive load, only shape and greyscale value are used. In this visualisation time scale is not used any more: only the order of states/transitions/alternatives is respected. Accordingly, durations and densities available in multi-hypothesis chronology diagrams cease to be readable. This visualisation focuses on relations of transitions to chronology and clues. Accordingly, other symbols (not present in the example given above) may be used to position specific events or processes such as archaeological surveys, reuse of parts of the artefact, etc.

The above graphs of potential interactions help visualising what an analyst does with each and every clue he knows of, including well-established indications. Accordingly, the resulting graphics may be lengthy, and appeared rather ill-suited to a synthetic comparison of levels of complexity of various artefacts. We therefore developed a third visualisation, called visual measure of complexity, in which we withdraw from the visualisation redundant well-established indications. The diagram focuses in focusing on alternative paths, and may be used as a visual indicator of complexity for a given artefact. Its main role for us is to foster comparisons across the collection. It is designed to underline different patterns of complexity - spatial, temporal, and semantic. Figure 7 for instance shows relative patterns of complexity of artefacts classified by what [4] calls social use.

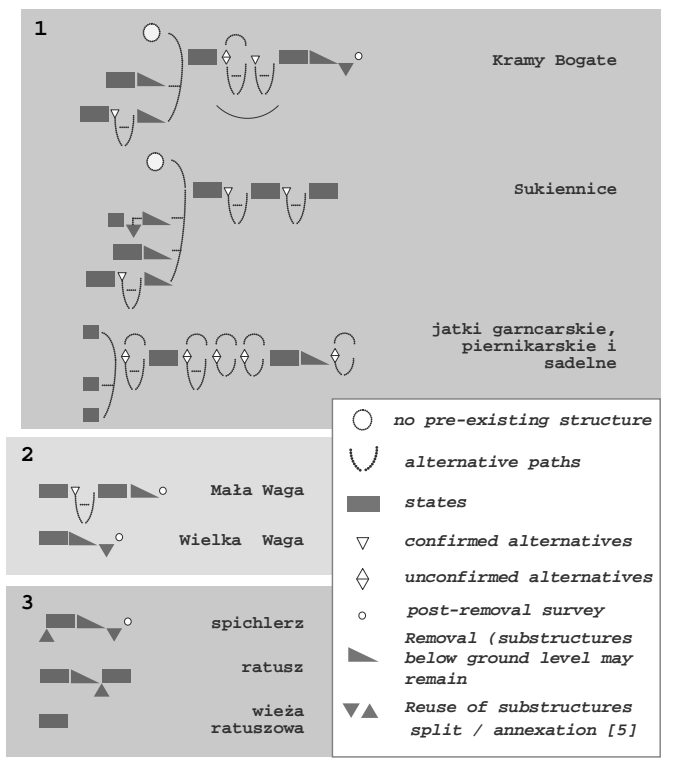

Figure. 7. Relative patterns of complexity for artefacts (1) dedicated to private or semi-private trading; (2) dedicated to 
collective administration of trading (3), dedicated to the administration of the town. Note on the bottom most line the simplicity of the diagram for Krakow's belfry. For this particular artefact we have spotted 28 successive transformations (a record across the collection). However since each of these transformations is rather well documented, and does not call for interpretation, the artefact's visual measure of complexity is limited to one rectangle.

\subsection{Visualising alternative scenarios in time and space across the collection of artefacts}

The visual measure of complexity can be seen as a first step in trying to analyse, beyond individual artefacts, the collection as a whole. However it only supports complexity reading, and anyway remains a solution putting "side by side" individual cases. Considering we studied a total of 27 artefacts, Tufte's recommendation "enforce comparisons within the eyespan" might not be easy to achieve. Accordingly, we have evaluated various visual solutions aimed at summing up in an abstract manner all changes and alternatives for each artefact. Solutions like mosaic plots or time series were fist tested but turned out hard to read because of the number of cases, and of their variety in terms of life span.

We here consider that alternatives about the initial phases of an artefact's creation, before a first confirmed indication can be quoted to say that it did exist, raise specific issues (in particular a long and very imprecise time slots, and a complex combinatory of possibilities that in theory could be extended further on). As a side-effect, doubts can be over-emphasized at visualisation time In the following graphics we therefore explicitly differentiate in the visualization:

- initial phases (represented by as many circles as there are possible initialisation points)

- confirmed and unconfirmed alternatives once the existence of the artefact is clearly established, counted and represented as known changes are but with specific colours.

We decided for a visualisation inspired by the "small multiples" concept in which we report for each artefact, the life span and the number of changes and of alternatives. The size of each square unit represents maximum values for these two parameters inside the collection (maximum life span is 1001 years - St Adalbert's church; maximum number of changes and alternatives is 28 - the belfry). Figure 8 sums up the content of a graphic unit.

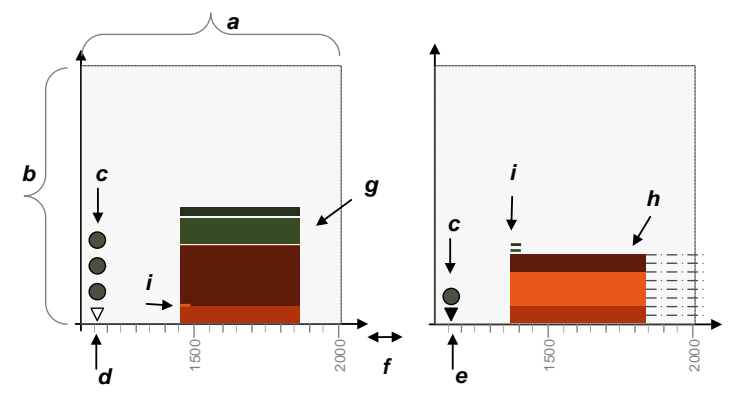

Figure. 8. A visualisation of the number and types of changes (vertical reading); and of the periods of existence of the artefacts (location in time and duration, horizontal reading). (a) the width of the square represents the maximum life span for our collection - 1001 years. (b) the height of the square represents the maximum number of changes inside our collection - 28 changes and alternatives. (c) circles representing the number of alternative initialisation points. (d) a white triangle stating an archaeological survey was carried out. (e) a black triangle stating that parts of the artefact are now reused inside another artefact, with a new function (f) the $x$ axis presents dates in real scale, and therefore allows the reading of absolute values for duration and time localisation (g) greenish rectangles represent confirmed (bottom) and unconfirmed (top, darker) alternatives. On user demand a background scale and/or the actual number can be superimposed- here two confirmed, one unconfirmed (h) reddish rectangles represent (bottom to top), morphological changes, episodic changes, destructive changes (i) Zeros represented by tiny lines.

Once a unit has been computed for each element in the collection, various combinations can be tried out to grab an understanding of the collection and spot temporal, spatial or other patterns. Figure 9 (durations) illustrates Bertin's vision of “graphics as an answer to a question", by showing no correlation can be established between the length of an artefact's life span, and the number of alternative scenarios of evolution.

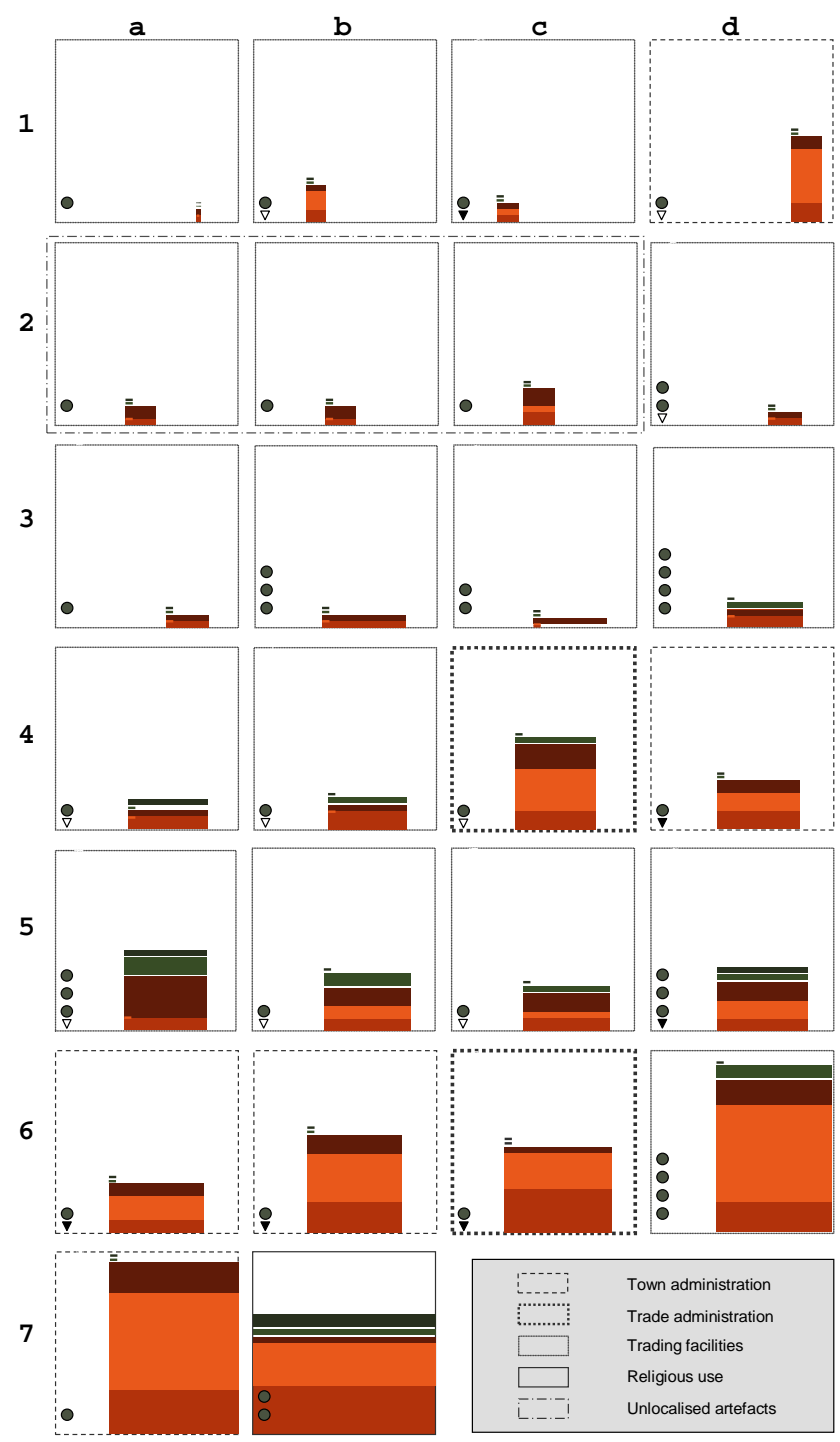

Figure. 9. A combination of graphic units corresponding to the 27 artefacts in our collection (classified by progressive lengths of life spans) with outlines of each unit corresponding to social 
uses. The visualisation corroborates some intuitive hypotheses: religious $(b 7)$ or administrative artefacts $(a 6, b 6, c 6$, and a7) appear as lasting longer, with less alternative scenarios. It also underlines exceptions to this pattern: (d6) unit corresponding to the cloth hall - a long history, with several alternative scenarios, and a maximum value for alternative initialisation points.

But the above visualisation still puts individual cases side by side. Accordingly we developed a solution this time capitalizing on the concept of time series (Figure 10). On the $\mathrm{x}$ axis are distributed even periods of time (50 years) starting from the foundation of the city in 1257 . On the y axis, we represent the overall amount of changes and alternatives for the whole collection are counted at each period. Circles that represent the overall amount of alternative initialisation points for each 50 years period are added below the $\mathrm{x}$ axis. The resulting figure does call a number of interesting remarks, summed up in the legend.

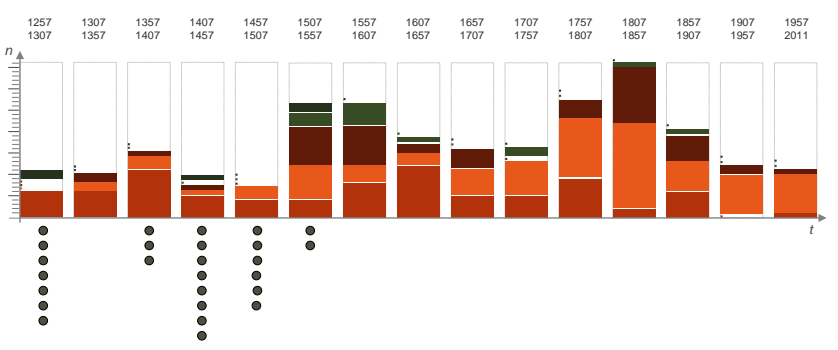

Figure. 10. The distribution of changes, alternatives and alternative initialisation points over time - with a fifty years time granularity (graphic codes are the same than for the previous figures). Note, unsurprisingly, that initialisation points concern early periods, but also, more surprisingly, that none are present in the 1307-1357 period, and that the maximum number of alternative initialisation points correspond to a period of low activity intensity (column 4). The visualisation also shows a sudden increase in density of changes at the beginning of the 16th century, with during the 1507-1557 period a clear majority of destructive changes (dark red) and in the following periods a majority of morphological changes - an indication of the duration of transformations at that time. An interesting point is also made concerning the first half of the 19th century (1807-1857). The period is known as the period during which most of the artefacts standing in the market square will be destroyed. But the visualisation shows that episodic changes widely outnumber destructive changes. This indicates that before deciding to tear down these artefacts, a number of solution were tried out to repair, transform, reuse them. In other words it clearly shows that the well-established "pattern of destruction" should rather be called "pattern of renunciation" in front of maintenance and repair difficulties. Finally, the visualisation underlines some clear patterns in the time distribution of alternative scenarios (green rectangles). Note for instance their density during the 16th century, or their presence even during the supposedly well-documented 19th century.

\section{IMPLEMENTATION, EVALUATION}

This development complements previous works on the same test field - the medieval heart of Kraków [5], [23]. Accordingly, the technical platform is here the same:
- a description of artefacts as instances of a hierarchy of classes (in the sense of OOP), with persistence enabled through RDBMS structures,

- information sets structured and annotated through RDBMS structures,

- dynamic outputs (may they be visual outputs - 3D VRML or 2D SVG- or textual outputs -XML) produced by Perl scripts,

- interfaces produced by Perl scripts either as XHTML (in our first experiments) or as XML/XSLT datasheets,

The whole system is flexible to incremental data update. It has to be mentioned here that graphics illustrating this paper are redrawn from the SVG outputs. The implementation is as can be seen a rather basic one, and calls no particular comment in the context of this paper.

One point must however be made clear: how graphic units can be distributed according to their relative geographical position. Because the contour of artefacts varies over time (and therefore their barycentres), and because we cannot even localise some of them (3 out of 27), sorting out artefacts using geometric rules of orientation, size or whatsoever appeared to be a dead-end. We therefore chose not to rely on an automatic procedure for the distribution of the graphic units. We implemented a semiautomatic procedure through which the user affects to each artefact a [line, column] position inside a predefined grid. These indications are written inside an ASCII configuration file that is interpreted on the fly when writing the SVG output. This unique, easy to understand, procedure can be adopted not only to locate graphic units with regards to their geographical position, but also to organise any semantically-consistent connections (social use, ownership, internal distribution of space, etc.). The solution might not be called a skilful one as far as computer science is concerned. Still, it is a convenient, robust one, and furthermore it nicely illustrates how puzzling it can be to try and bridge the gap between the systematic approach a developer favours, and the craftsman approach our information sets sometimes dictate.

Although the various choices we made, in particular on graphic aspects, were intersubjectively evaluated on a regular basis all along this research (sending a great number of proposals to the delete zone), we did not take the time to formalise a structured evaluation. The visualisations, applied to Kraków’s Rynek Główny, helped us reach some interesting conclusions on this case study. They for instance clearly deny "common sense" assumptions such as "the more information the less doubts" or "the most recent the most well established", etc. But an outside view on this development now needs to be called in. This is clearly a weakness of the proposal at this stage. An evaluation is programmed through which we shall investigate two different issues:

- To which extent do the graphics leave room for ambiguities and misinterpretations, and to which extent do they foster knowledge sharing? This will be done by questioning nonexperts on readability, memorisation, exception spotting and cognitive load issues.

- Is the framework acceptable as it is for people whose professional practices do not always include a strong investment in computer technologies in general, and in data visualisation in particular (namely, historians, historians of art, archaeologists, etc.). This will be done, in collaboration with colleagues who have thoroughly analysed some of the case studies, by analysing how alternatives spotted in this research match their doubts. 


\section{CONCLUSION}

This research aimed at providing analysts of historic architecture with means to visualise interpretation difficulties, i.e. visualise alternative scenarios that can be derived from sets of information. We have briefly commented on the nature of their cognitive process - hypo-deductive, reductive, non-inductive. We have shown that it is necessary to try and support this process with graphics highlighting alternative explanations so as to avoid unjustified assumptions. We have analysed factors that need to be weighed when interpreting the underlying sets of information, and developed various visual solutions:

- multi-hypothesis chronology diagrams, that deliver quick, synthetic view on where alternatives exist (in time) and on what options the analysts face;

- graphs of potential interactions, through which we introduce a classification of architectural consequences. These graphs position alternatives in time, help understanding their relative density when compared to well-established indications, and connect them to the corresponding clues;

- visual measures of complexity, a diagram that fosters comparisons of the relative complexity of artefacts evolution across the collection.

- Visualisations dedicated to collection reading in which we report for each artefact, the life span and the number of changes and of alternatives.

The results we report show that a number of faulty inferences can be fruitfully uncovered by analytical graphics - provided these graphics do take into consideration the nature of the data handled in historical sciences, and the nature of the analyst's cognitive process. Although insufficiently evaluated at this stage, we believe the contribution draws attention on the necessity to further investigate interpretation steps in historic architecture, and more generally on the complexity of the challenging complexity of simple visual thinking. But we consider that one of the contribution's main achievements is to show that analysts in historical sciences - often driven towards solutions stemming from geosciences - can greatly benefit from an investment in the infovis community.

\section{ACKNOWLEDGMENTS}

The authors wish to thank W. Komorowski and T. Węcławowicz for their feedback and suggestions.

\section{REFERENCES}

[1] Gershon, N.D. 1998. Visualization of an imperfect world. In IEEE Computer Graphics and Applications vol. $18 n^{\circ} 4$.

[2] Johnson, I. 1999. Mapping the fourth dimension: the TimeMap project. In Archaeology in the Age of the Internet. Dingwall, L, Ed. British Archaeological Rep. Int. Series 750.

[3] Van Ruymbeke, M, et al. 2008. Development and use of a 4D GIS to support the conservation of the Calakmul site. In Proc. 1st International EARSeL Workshop, Lasapona, $\mathrm{R}$ Masini, N (Eds) Aracne.

[4] Rodier, X, Saligny, L. Lefevre, B. 2008. Understanding urban fabric with the OH_FET model based on socialuse, space and time. In online journal Archeologia $e$ Ccalcolatori; <http://soi.cntds.it/archcalc>

[5] Dudek,I, Blaise, J.Y 2008. Visual assessment of heritage architecture life cycles. In Proceedings of the 8th
International Conference on Knowledge Management IKnow 2008, I-Know I-Media 08, (Graz, Austria, Sept 2008) JUCS, ISSN 0948-695x.

[6] Delattre, P. 1974. Concepts de formalisation et concepts d'exploration, In Scientia (rivista di scienza), Milan.

[7] Tufte, E.R., 1990-2001. Envisioning information. (alternative explanations) Graphics Press, Cheshire.

[8] Dudek,I. , Blaise, J.Y 2006. Informative Modelling. In MiaJournal, Vol. 1s.

[9] Zhao, J. et al., 2008. Activities, ringmaps and geovisualization of large human movement fields. In Information visualization Volume $7 \mathrm{~N}^{\circ} 3$, Palgrave.

[10] Knopf, G.K et al., 2002. Representing high-dimensional data sets as closed surfaces. In Information visualization Volume 1 $N^{\circ}$ 2, Palgrave.

[11] Sanders, L 2007 (Ed) Models in Spatial Analysis, London ; Newport Beach, CA : ISTE.

[12] Chardonell, S 2007 Time-Geography: individuals in time and space. In Sanders, L Models in Spatial Analysis, (op.cit).

[13] Hagen-Zanker, A., 2008. Visualization and classification of urban change patterns on the basis of state-space transitions. In 11th Int. Conf. on Geographic Information Science AGILE 2008 (Girona, Spain) LNGC Springer-Verlag, Heidelberg.

[14] Kienreich, W. 2006. Information and Knowledge Visualisation: an oblique view, In Proceedings MIA workshop, (Saint-Maximin, France, june 2006), MiaJournal, Vol. 0.

[15] Bocheński, J.M. 1968 The methods of contemporary thought Harper \& Row, New York.

[16] Neth, H., Beller, S. 1999. How knowledge interferes with reasoning - Suppression effect by content and context. In Proceedings of the 21st Annual conf. of the Cognitive Science Society M.Hahn \& S.C. Stoness (Eds.) Mahwah, N.J.

[17] Thomson,J., Hetzler,B., MacEachren, A., Gahegan, M., Pavel, M. 2005. A typology for visualizing uncertainty. In Proceedings of the conf. on Visualization and Data analysis 2005 (San Jose, CA, jan 2005), SPIE proc. series, vol 5669.

[18] Bertin, J; 1967/1998. Sémiologie graphique, Editions EHESS, Paris.

[19] Friendly, M 2006. A brief history of data visualization. In handbook of computational statistics: data visualization. C.Chen W.Hardle A.Unwin (Eds.) Springer-Verlag, Heidelberg.

[20] Rød, J.K 2003. The third choice. In Cybergeo, European journal of geography; < http://cybergeo.revues.org/1625>

[21] Sabol, V., Scharl, A. 2008. Visualizing Temporal-Semantic Relations in Dynamic Information Landscapes. In 11th Int. Conf. on Geographic Information Science AGILE 2008 (Girona, Spain) LNGC Springer-Verlag, Heidelberg.

[22] Seifert, M, Sabol, V, Kienreich, W, 2010 Stress Maps: analysing local phenomena in dimensionality reduction based visualisations. In IEEE Int. symposium on Visual Analytics Science and Technology. Kolhammer, J, Keim, D (Eds).

[23] Dudek,I, Blaise, J.Y 2010. Understanding changes in historic architecture. In Proceedings IMAGAPP/IVAPP 2010 (Angers, France) INSTICC. 